\title{
A SEARCH FOR LOW-LEVEL SEYFERT ACTIVITY IN THE 500 BRIGHTEST NORTHERN GALAXIES*
}

\author{
Alexei V. Filippenko \\ Department of Astronomy, University of California \\ Berkeley, CA 94720 USA \\ Wallace L. W. Sargent \\ Palomar Observatory, California Institute of Technology \\ Pasadena, CA 91125 USA
}

\begin{abstract}
A sensitive search for intrinsically faint Seyfert nuclei in the $\mathbf{5 0 0}$ brightest northern galaxies is being conducted with the Hale $5.08 \mathrm{~m}$ telescope. The primary aim is to extend the luminosity function of active galactic nuclei (AGNs) to lower luminosities. Thus far, we have detected $\mathrm{H} \alpha$ emission having full-width near zero-intensity (FWZI) $\gtrsim 4000 \mathrm{~km} \mathrm{~s}^{-1}$ in a significant fraction ( $\gtrsim 10 \%$ ) of nearby galaxies. Moreover, the narrow emission lines in a given nucleus often have markedly different widths, as shown in detail for M81. Those associated with high critical densities are generally the broadest, indicating that a very wide range of densities is present in the narrow-line region. The relative intensities of the lines can readily be explained with models in which clouds of gas having $n_{e} \approx 10^{2}$ to $10^{7} \mathrm{~cm}^{-3}$ are photoionized by nonstellar radiation such as that produced by QSOs and luminous Seyfert 1 galaxies.
\end{abstract}

\section{INTRODUCTION}

There is considerable evidence for continuity between the properties of QSOs and classical Seyfert galaxies. This continuity is not restricted only to the luminosities and spectral characteristics (Weedman 1977) of the active central regions; deep images of low-redshift QSOs $(z \lesssim 0.4)$ almost always reveal nebulosities whose sizes, morphologies, and colors resemble those of the relatively normal galaxies which harbor Seyfert nuclei (Malkan 1984, and references therein). In several cases, high-quality spectra have directly shown that starlight accounts for a substantial portion of the extended "fuzz" (e.g., Boroson, Oke, and Green 1982). It is likely, therefore, that we are witnessing the same basic phenomenon, such as the accretion of matter by a supermassive black hole (see Rees 1984), at many different levels of vigor in these objects. The brightest QSOs $\left(-31 \lesssim M_{V} \lesssim-27\right)$ represent one extreme in absolute luminosity, although fainter QSOs $\left(-27 \lesssim M_{V} \lesssim\right.$ $-23)$ and Markarian Seyfert nuclei $\left(-23 \lesssim M_{V} \lesssim-19\right)$ are more numerous. Even milder activity can be found in the nuclei of nearby galaxies such as NGC 4051 $\left(M_{V} \approx-15.6\right.$; Véron 1979; $\left.H_{0}=50 \mathrm{~km} \mathrm{~s}^{-1} \mathrm{Mpc}^{-1}\right)$.

* Research based on observations made at Palomar Observatory, Caltech. 
But just how quiescent can this "activity" in the nuclei of galaxies be? Is there a fundamental limit below which the mechanisms responsible for the nonstellar continuum, broad emission lines, and violent jets cannot operate? Do the nuclei of galaxies frequently support activity at the lowest allowed level, or are such objects as rare as the very brightest QSOs? Did they begin their lives as intrinsically luminous beacons which gradually faded with time?

In essence, what we need to determine is $\Phi(L, z)$, the complete luminosity function of AGNs at all epochs; only then will we be able to answer with reasonable certainty these and other important questions. From a practical point of view, of course, this is an impossible task with currently available instruments. We can, however, derive $\Phi(L, 0)$, the complete luminosity function at the present epoch, and compare it with the partial information obtained for higher redshifts. The high and intermediate-luminosity portions of $\Phi(L, 0)$ are already well known (e.g., Schmidt and Green 1983; Meurs and Wilson 1984; Marshall 1985). Intrinsically faint AGNs, on the other hand, are difficult to find because they are usually dwarfed by their host galaxies; knowledge of their space density is quite limited. However, optical surveys such as those of Stauffer (1982), Keel (1983), and Phillips, Charles, and Baldwin (1983), as well as radio (Sadler 1987) and X-ray (Maccacaro 1987) investigations, suggest that they are common.

During the past two years we have been engaged in a major observational effort to discover and study all nearby, low-luminosity ("dwarf") AGNs; see Filippenko and Sargent (1985, 1986a - Papers I and II). Spectra of every galaxy with $\delta \geq 0^{\circ}$ and $B_{T} \leq 12.5$ are being obtained, and a careful search is made for Seyfert-like activity. It is assumed that a Seyfert 1 nucleus is characterized by the presence of $\mathrm{H} \alpha$ emission, no matter how faint, which is substantially broader than strong forbidden lines such as [O III] $\lambda 5007$ and [N II] $\lambda 6583$; typically this means $\mathrm{FWZI}(\mathrm{H} \alpha) \gtrsim 4000 \mathrm{~km} \mathrm{~s}^{-1}$. The relative intensities of the narrow emission lines, often the only ones visible, constitute secondary criteria that can be used to distinguish between weak Seyfert 2 nuclei, "low-ionization nuclear emission-line regions" (LINERs; Heckman 1980), H II regions, and other types of objects. In the next section we briefly describe the observations and some of our results.

\section{THE SURVEY}

Roughly 500 galaxies satisfy our selection criteria, and spectra of $\sim 400$ of them have already been obtained. The reduction and analysis of the data, however, are far from complete; thus, no statistical conclusions have been drawn.

The Double Spectrograph (Oke and Gunn 1982) at the Cassegrain focus of the Hale $5.08 \mathrm{~m}$ reflector at Palomar Observatory is being used to obtain the spectra through a long slit of width $2^{\prime \prime}$ (and sometimes $1^{\prime \prime}$ ). A dichroic filter directly behind the slit reflects blue light $(\lambda \lesssim 5500 \AA)$ to one grating and transmits red light to another. Integration times of $20-40$ minutes yield red spectra usually having signal-to-noise ratios of $\sim 100 / 1$ in the nucleus. The blue spectra are of lower quality, primarily because the galaxies are fainter, and the detector is less sensitive, at shorter wavelengths. In addition, the observations are generally made during times of bright moonlight, when the sky is very blue. The two spectral ranges are $\sim \lambda \lambda 4220-5090$ and $\sim \lambda \lambda 6210-6860$, with resolutions (FWHM) of $\sim 4.0-4.5 \AA$ and $\sim 2.0-2.6 \AA$, respectively. Since the slit is placed across the nucleus and the data are recorded on two-dimensional CCDs (charge-coupled devices), we are also able to determine rotation curves, abundance gradients, 
radial variations in emission-line intensities and widths, and other properties of interest. Papers I and II describe the procedures used to manipulate the spectra. An excellent example of a low-luminosity Seyfert 1 nucleus is that of NGC 4639 in the Virgo cluster (see Fig. 3 of Paper II). The broad $\mathrm{H} \alpha$ emission line $\left(F W Z I \approx 8600 \mathrm{~km} \mathrm{~s}^{-1}\right.$ ) is very prominent, but its absolute intensity is much less than in classical type 1 Seyferts. Figure 1 illustrates NGC 4579, an Sab galaxy with an even fainter active nucleus. The $\mathrm{H} \alpha$ emission line exhibits broad wings $\left(F W Z I \approx 5700 \mathrm{~km} \mathrm{~s}^{-1}\right.$; Paper I) which were first noticed by Stauffer (1982) and confirmed by Keel (1983). Although the exact extent of the line is difficult to measure, the task becomes much easier after subtraction of the underlying starlight (see below). A major portion of the wings is almost certainly not due to [N II] emission, since other forbidden lines in the red and blue spectra lack such wings. NGC 4579 is a classical LINER; [O I] $\lambda 6300$ and [S II] $\lambda \lambda 6716,6731$ are strong in comparison with [O III] $\lambda 5007$.

Somewhat less obvious broad wings attributable to $\mathrm{H} \alpha$ can be found in the spectrum of NGC 1052 (Fig. 2), an E3/S0 galaxy which is formally outside the limits of our survey but was observed because of its great historical importance in the study of LINERs. The narrow emission lines all have quite symmetrical, nearly Gaussian profiles, yet the $\{\mathrm{H} \alpha+[\mathrm{N}$ II $]\}$ blend has excess emission on

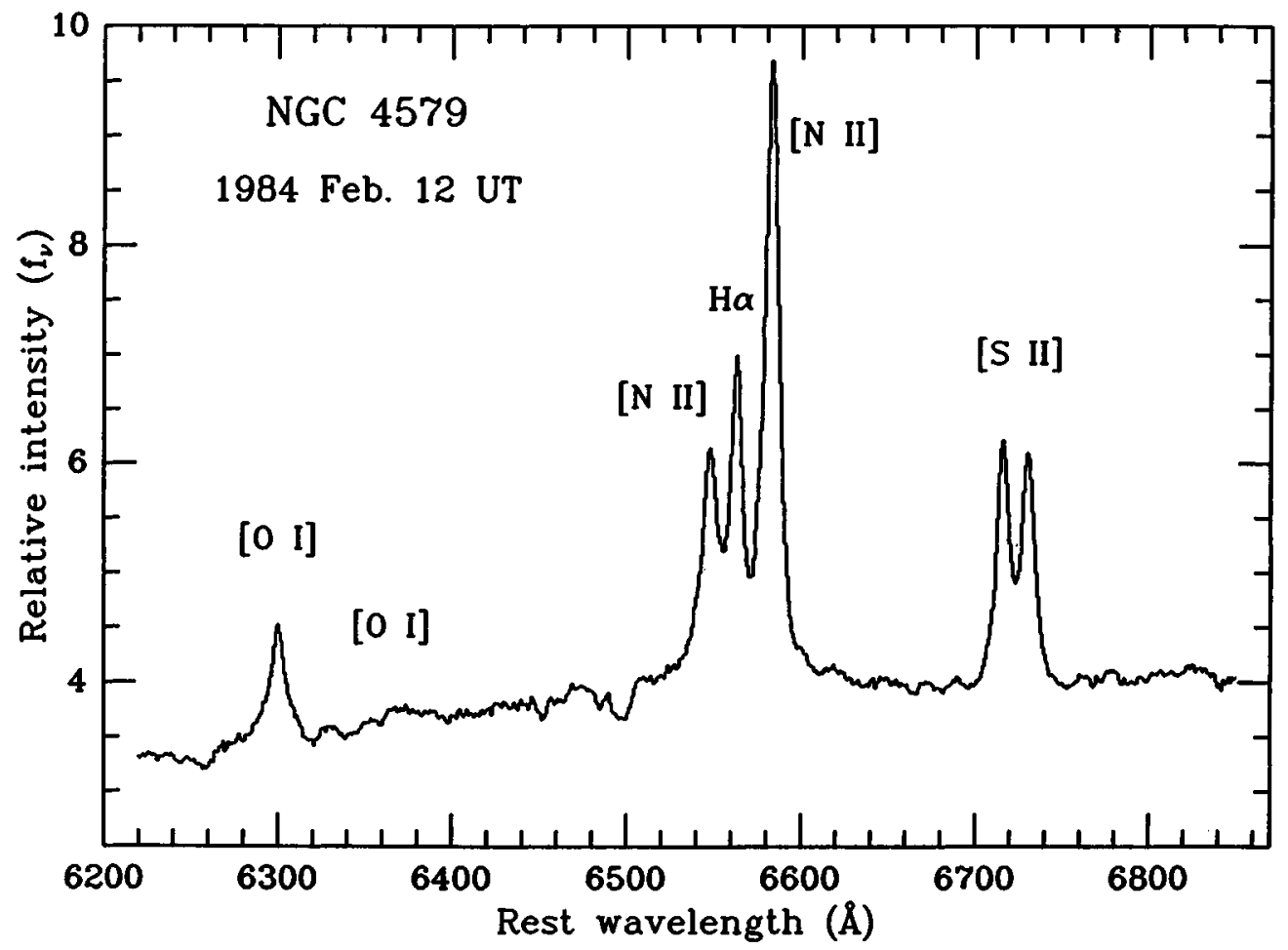

Figure 1: Red spectrum of the nucleus of NGC 4579. Faint, broad wings are visible in the $\{\mathrm{H} \alpha+[\mathrm{N} \mathrm{III}]\}$ blend; these are attributed to $\mathrm{H} \alpha$. Absorption lines from an old stellar population are present, and seriously alter the apparent strengths of some emission lines (e.g., [O I] $\lambda 6364$ ). 
both the red and blue sides. Wings like these are absent in the profiles of other forbidden lines in NGC 1052, so they are probably associated with $\mathrm{H} \alpha$ (FWZI $\gtrsim 6000 \mathrm{~km} \mathrm{~s}^{-1}$ ) rather than [N II]. Similar features were found in many of the galaxies analyzed in Paper I, perhaps most notably M87 (see Fig. 5 in Paper II).

Thus, NGC 1052 harbors a low-luminosity Seyfert 1 nucleus which, by analogy with classical AGNs, probably emits a weak ionizing continuum. This result is particularly important in light of the recent emphasis on explaining the relative intensities of the narrow lines in LINERs with photoionization models characterized by low values of the ionization parameter (e.g., Ferland and Netzer 1983; Halpern and Steiner 1983; Binette 1985), instead of with shock models (e.g., Koski and Osterbrock 1976; Fosbury et al. 1978; Heckman 1980). Over one-third of the LINERs studied in Paper I also have detectable broad $\mathrm{H} \alpha$ wings, reinforcing the idea that low-level QSO activity is present in their nuclei. Of all the galaxies we have observed to date, roughly $10 \%$ are type 1 Seyferts according to our definition.

The skeptic may contend that the features we are measuring are so weak that their reality is dubious. Careful perusal of Figure 2 in Paper II, however, shows that two spectra of the Sa galaxy NGC 2681, obtained at different times

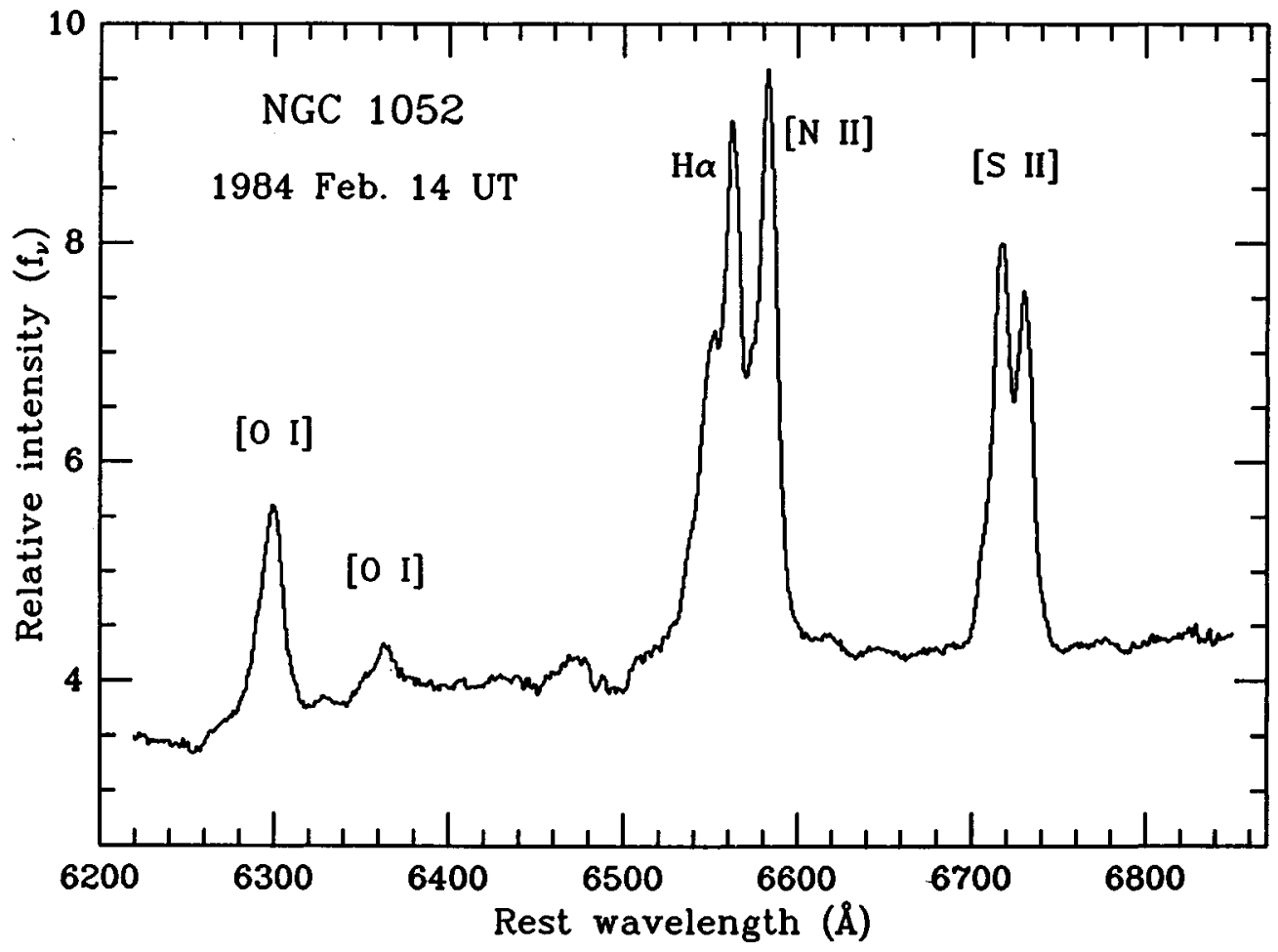

Figure 2: Relatively broad emission lines of neutral and singly ionized atoms are very prominent in the nucleus of NGC 1052, the prototypical LINER. The $\{\mathrm{H} \alpha+[\mathrm{N} \mathrm{III}\}$ blend exhibits weak wings that are probably due to a very broad component of $\mathrm{H} \alpha$ emission similar to, but much fainter than, that in "classical" type 1 Seyfert galaxies. 
and on different locations of the CCD, are almost indistinguishable. Figure 12 of Paper II illustrates the same thing for M81; even the most minute undulations appear the same in both spectra. But perhaps the most convincing evidence is that spectra of different galaxies having similar metallicity and stellar velocity dispersion are also nearly identical. This is illustrated in Figure 3, where a spectrum $(a)$ of M81 obtained through a $1^{\prime \prime}$ slit (resolution $\approx 1.7 \AA$ ) is compared with the spectrum (b) of an absorption-line "template" galaxy, NGC 4339. The latter was normalized with a quadratic polynomial to force the intensity and overall continuum shape to be similar to that of M81.

The difference between $(a)$ and $(b)$ is shown in Figure 3c. Almost no trace of the stellar continuum remains in spectral regions devoid of emission lines; the same is therefore likely to be true elsewhere as well. Faint emission lines such as [O I] $\lambda 6364$ and $\mathrm{He}$ I $\lambda 6678$ can now be measured accurately. Note, in particular, the great extent of the broad $\mathrm{H} \alpha$ emission line, which was discovered by Peimbert

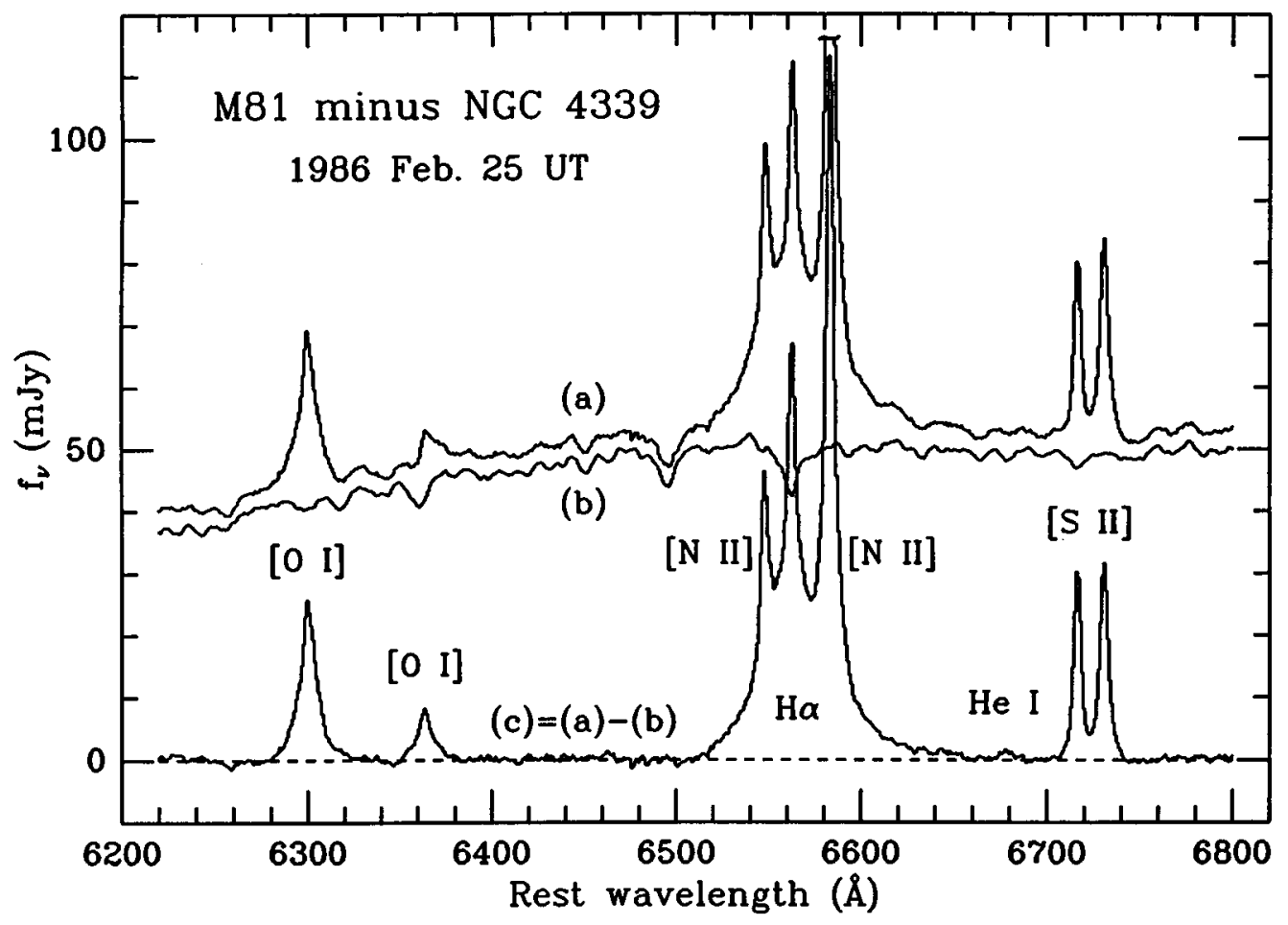

Figure 3: (a) Flux-calibrated red spectrum of the nucleus of M81. A suitably scaled spectrum of an absorption-line template galaxy, NGC 4339, is illustrated in (b), with an offset of $-3 \mathrm{mJy}$ from $(a)$ for clarity. The difference between these two, (c), represents the pure emission-line spectrum of M81. Note the presence of He I $\lambda 6678$, which was not visible in $(a)$, and the extended red wing of the broad $\mathrm{H} \alpha$ emission line. Other emission lines affected by stellar absorption include $[\mathrm{O} \mathrm{I}] \lambda 6364, \mathrm{H} \alpha$, and [S II] $\lambda 6716$. The [O I] lines are much broader than [S II] and [N II], and appear to have nearly Lorentzian, rather than Gaussian, profiles. [S II] $\lambda 6731$ is roughly $20 \%$ broader than [S II] $\lambda 6716$. 
and Torres-Peimbert (1981) and confirmed by Shuder and Osterbrock (1981). The line intensity ratios and profiles can be used in various models to determine the excitation mechanism and other properties of the nucleus. This procedure, or similar ones, is applied to all galaxies in which underlying starlight severely contaminates the emission lines.

\section{THE NUCLEUS OF M81}

In searching for significant correlations and clues to the intrinsic characteristics of galactic nuclei, it is often best to initially concentrate on extreme examples. M81 provides a beautiful case in point, which we will now discuss. It is described in detail by Filippenko and Sargent (1986b).

Figure $3 c$ illustrates an important property found among many of the LINERs and dwarf Seyfert nuclei in our survey: the widths and profiles of the narrow lines are not identical. It is abundantly clear that [O I] $\lambda 6300$ is far broader than the $[\mathrm{N} \mathrm{II}]$ and $[\mathrm{S} \mathrm{II}]$ lines, and its profile is distinctly non-Gaussian. The wings have FWZI $\approx 2100 \mathrm{~km} \mathrm{~s}^{-1}$, a value intermediate between that of the [S II] lines $\left(\sim 950 \mathrm{~km} \mathrm{~s}^{-1}\right)$ and the broad $\mathrm{H} \alpha$ emission $\left(\sim 6700 \mathrm{~km} \mathrm{~s}^{-1}\right)$. The same can be seen to a lesser extent in NGC 1052 (Fig. 2). As discussed by Filippenko and Halpern (1984), Filippenko (1985), and in Papers I and II, this indicates that the narrow-line region (NLR) is composed of clouds spanning a wide range of gas densities $\left(n_{e} \approx 10^{2}-10^{7} \mathrm{~cm}^{-3}\right)$, and that their bulk motion increases with increasing density. A given emission line is preferentially produced by gas whose density is close to the critical density for collisional deexcitation [ $n_{e}$ (crit)] of the corresponding atomic transition. The [O I] $\lambda 6300$ line, for example, has $n_{e}$ (crit) $\approx 1.4 \times 10^{6} \mathrm{~cm}^{-3}$, whereas the much narrower [S II] $\lambda \lambda 6716,6731$ lines have $\left\langle n_{e}(\mathrm{crit})\right\rangle \approx 2.1 \times 10^{3} \mathrm{~cm}^{-3}$.

It is interesting to note that slight differences in line width are perceptible even in the individual [S II] lines themselves (Filippenko and Sargent 1986b): [S II] $\lambda 6716$ has FWHM $\approx 220 \mathrm{~km} \mathrm{~s}^{-1}$ and $n_{e}$ (crit) $=1.2 \times 10^{3} \mathrm{~cm}^{-3}$, whereas [S II] $\lambda 6731$ has FWHM $\approx 260 \mathrm{~km} \mathrm{~s}^{-1}$ and $n_{e}$ (crit) $=3.1 \times 10^{3} \mathrm{~cm}^{-3}$ ! Furthermore, the intensity ratio of [S II] $\lambda 6731$ to [S II] $\lambda 6716$ approaches the high-density limit $(\sim 2.5)$ in the line wings, so that $n_{e} \gtrsim 5 \times 10^{4} \mathrm{~cm}^{-3}$, whereas $n_{e} \lesssim 8 \times 10^{2}$ $\mathrm{cm}^{-3}$ in the line core. This once again shows that dense clumps of gas move most rapidly. Such differences have never before been reported for the red [S II] doublet, but in fact they are visible in Figure 12 of Paper II.

By analogy with the broad-line and narrow-line regions of AGNs, the gas density in the NLR itself probably increases monotonically with decreasing distance from the exact galactic center (Filippenko and Halpern 1984). In M81, this radial gradient of density and velocity is particularly steep. A number of Seyfert galaxies also exhibit a correlation between $n_{e}$ (crit) and line width (e.g., Pelat, Alloin, and Fosbury 1981; De Robertis and Osterbrock 1984; Carswell et al. 1984). In the most favorable objects, it may be possible to use the Hubble Space Telescope to spatially resolve the "intermediate" zone, whose density is $\sim 10^{6} \mathrm{~cm}^{-3}$, from regions in which $n_{e} \approx 10^{3} \mathrm{~cm}^{-3}$ or $n_{e} \approx 10^{9} \mathrm{~cm}^{-3}$.

Although standard emission-line diagnostics long ago showed that a range of densities must exist in the NLRs of many classical AGNs (e.g., Baldwin 1975; Osterbrock, Koski, and Phillips 1976; Neugebauer et al. 1976; Oke 1978), numerous single-density photoionization models have been constructed over the past 
decade because of their relative simplicity. Recently, however, the inadequacy of even the most sophisticated codes in reproducing the integrated emission-line intensity ratios has been emphasized by Péquignot (1984), Stasińska (1984), and others. The large differences in line profiles observed in M81 and other galaxies provide direct evidence for a "stratified" NLR, and reinforce their conclusion that single-density models are grossly unrealistic.

A few multi-density photoionization models have now been constructed, and the calculated intensity ratios agree quite well with observations. In particular, the unexpectedly large ratio of auroral lines (such as [O II] $\lambda \lambda 7319,7330$ ) to the corresponding nebular lines ([O II] $\lambda \lambda 3726,3729)$ is fully explained (Péquignot 1984; Filippenko and Halpern 1984), as is the great strength of [O I] $\lambda 6300$ (Filippenko 1986). Of course, heating by shocks may still play a significant role in some objects, and should therefore be included in the most complete calculations (Aldrovandi and Contini 1984).

\section{ACKNOWLEDGMENTS}

We thank the Time Allocation Committee and the staff of Palomar Observatory for their constant support of this long-term project, which is being funded by NSF grant AST 84-16704 (W. L. W. S.) and the Miller Institute for Basic Research in Science (A. V. F.). The financial assistance of the Armenian Academy of Sciences, as well as an AAS International Travel Grant from the NSF, made it possible for A. V. F. to attend IAU Symposium 121. The hospitality of the Byurakan Astrophysical Observatory, and especially of Professor E. Ye. Khachikian, is very gratefully acknowledged.

\section{REFERENCES}

Aldrovandi, S. M. V., and Contini, M. 1984, Astr. Ap., 140, 368.

Baldwin, J. A. 1975, Ap. J., 201, 26.

Binette, L. 1985, Astr. Ap., 143, 334.

Boroson, T. A., Oke, J. B., and Green, R. F. 1982, Ap. J., 263, 32.

Carswell, R. F., Baldwin, J. A., Atwood, B., and Phillips, M. M. 1984, Ap. J., 286, 464.

De Robertis, M. M., and Osterbrock, D. E. 1984, Ap. J., 286, 171.

Ferland, G. J., and Netzer, H. 1983, Ap. J., 264, 105.

Filippenko, A. V. 1985, Ap. J., 289, 475.

Filippenko, A. V. 1986, in Quasars, eds. G. Swarup and V. K. Kapahi (Dordrecht: Reidel), in press.

Filippenko, A. V., and Halpern, J. P. 1984, Ap. J., 285, 458.

Filippenko, A. V., and Sargent, W. L. W. 1985, Ap. J. Suppl., 57, 503 (Paper I).

Filippenko, A. V., and Sargent, W. L. W. 1986a, in Structure and Evolution of Active Galactic Nuclei, eds. G. Giuricin et al. (Dordrecht: Reidel), 21 (Paper II).

Filippenko, A. V., and Sargent, W. L. W. 1986b, Ap. J., submitted.

Fosbury, R. A. E., Mebold, U., Goss, W. M., and Dopita, M. A. 1978, M. N. $R$. A. S., 183, 549.

Halpern, J. P., and Steiner, J. E. 1983, Ap. J. (Letters), 269, L37.

Heckman, T. M. 1980, Astr. Ap., 87, 152. 
Keel, W. C. 1983 Ap. J., 269, 466.

Koski, A. T., and Osterbrock, D. E. 1976, Ap. J. (Letters), 203, L49.

Maccacaro, T. 1987, these proceedings.

Malkan, M. A. 1984, Ap. J., 287, 555.

Marshall, H. L. 1985, Ap. J., 299, 109.

Meurs, E. J. A., and Wilson, A. S. 1984, Astr. Ap., 136, 206.

Neugebauer, G., Becklin, E. E., Oke, J. B., and Searle, L. 1976, Ap. J., 205, 29.

Oke, J. B. 1978, J. R. A. S. Canada, 72, 121.

Oke, J. B., and Gunn, J. E. 1982, Pub. A. S. P., 94, 586.

Osterbrock, D. E., Koski, A. T., and Phillips, M. M. 1976, Ap. J., 206, 898.

Peimbert, M., and Torres-Peimbert, S. 1981, Ap. J., 245, 845.

Pelat, D., Alloin, D., and Fosbury, R. A. E. 1981, M. N. R. A. S., 195, 787.

Péquignot, D. 1984, Astr. Ap., 131, 159.

Phillips, M. M., Charles, P. A., and Baldwin, J. A. 1983, Ap. J., 266, 485.

Rees, M. J. 1984, Ann. Rev. Astr. Ap., 22, 471.

Sadler, E. M. 1987, these proceedings.

Schmidt, M., and Green, R. F. 1983, Ap. J., 269, 352.

Shuder, J. M., and Osterbrock, D. E. 1981, Ap. J., 250, 55.

Stasińska, G. 1984, Astr. Ap., 135, 341.

Stauffer, J. R. 1982, Ap. J., $262,66$.

Véron, P. 1979, Astr. Ap., 78, 46.

Weedman, D. W. 1977, Ann. Rev. Astr. Ap., 15, 69. 


\section{DISCUSSION}

WILSON: In Seyfert 1 galaxies, there is known to be a strong correlation between the $\mathrm{H} \alpha$ or $\mathrm{H} \beta$ power and the X-ray continuum power. When the X-ray power is weak, there are other processes (like binary X-ray sources, supernova remnants) that can dominate the $\mathrm{X}$-ray emission. Therefore, it would be very interesting to know whether the correlation continues to low X-ray luminosities, since it would suggest that the $\mathrm{X}$-ray process may be the same as in the high-luminosity galaxies. Do you have any comments?

FILIPPENKO: It would indeed be interesting to see if the correlation continues, and we plan to pursue this matter. Unfortunately, relatively few of the galaxies we are studying were detected with the Einstein Observatory. In constructing the $\mathrm{X}$-ray luminosity function of nearby galaxies, Elvis, Soltan, and Keel (1984, $A p$. $J ., 283,479)$ assumed that $\left\langle L_{X} / L_{H \alpha}\right\rangle=40$, as in QSOs and Seyfert 1 nuclei, but we will be able to check this directly for at least a few dozen objects.

WARD: How are you measuring the absolute magnitude in order to construct the luminosity function?

FILIPPENKO: We generally measure the flux of the broad $\mathrm{H} \alpha$ emission line through a $2^{\prime \prime} \times 4.1^{\prime \prime}$ aperture synthesized from the slit spectrum. Since the atmospheric seeing is monitored throughout the night, approximate correction factors can be applied to the $\mathrm{H} \alpha$ fluxes. The absolute strength of the nonstellar continuum is assumed to be proportional to that of $\mathrm{H} \alpha$, with the same scale factor as in classical Seyferts and QSOs.

Together with Kate Ebneter of U. C. Berkeley, we are also obtaining direct, photometric CCD images of each galaxy through two different filters, with the $1 \mathrm{~m}$ Nickel reflector at Lick Observatory. They will be used to measure the apparent brightness of each galactic nucleus through any desired aperture. In conjunction with broad-H $\alpha$ equivalent widths derived from the Palomar spectra, these data should provide relatively accurate $\mathrm{H} \alpha$ fluxes.

MELNICK: Have you checked that the weak, broad profiles of $\mathrm{H} \alpha$ are not due to instrumental effects?

FILIPPENKO: Yes. Many galactic nuclei exhibit very strong, narrow emission lines with no trace of broad wings. There are also a few galaxies with no emission lines whatsoever. Moreover, we have repeated the observations of several objects, and in all cases our original results were confirmed.

KOMBERG: Is there any correlation between the absolute nonstellar luminosity and the width of the broad permitted emission lines (e.g., $\mathrm{H} \alpha$ ) in AGNs?

FILIPPENKO: Over a wide range of absolute luminosities $\left(-31 \lesssim M_{V} \lesssim-16\right.$, and perhaps even down to $M_{V} \approx-11$ ), there is very little correlation. The 
brightest nuclei may have somewhat broader permitted lines than the faintest ones, but not by more than a factor of $\sim 3$.

KOMBERG: It seems unlikely, therefore, that the mass of the hypothetical black hole can be calculated by applying Kepler's law to the measured line widths. After all, we expect the high-luminosity objects to have black holes of greater mass than low-luminosity AGNs, so their emission lines should be much broader.

FILIPPENKO: Not necessarily. Many models (e.g., Krolik, McKee, and Tarter 1981, Ap. J., 249, 422) suggest that the broad-line clouds can form only over a very restricted range of ionization parameters. Since their gas density appears to be nearly the same in all AGNs, the clouds will be systematically farther from the nucleus in luminous objects than in faint ones. Hence, the greater mass of the black hole in luminous AGNs may be almost completely balanced by the larger distance to the clouds. Of course, it is also possible that the line widths are not predominantly of gravitational origin, in which case Kepler's law should not be used.

PASTORIZA: After subtraction of the stellar continuum, is there any evidence for a nonthermal contribution?

FILIPPENKO: The activity in most of the galaxies we are studying is so weak that it is not possible to detect the nonstellar continuum in our spectra. Differences in the stellar population and metallicity between the template galaxy and the low-luminosity active nucleus can easily mask the presence of a faint, featureless continuum. The continuum would undoubtedly be easiest to detect at extreme blue wavelengths $(\sim \lambda \lambda 3200-3600)$, but we have obtained very few spectra over this interval.

HUTCHINGS: Please comment on IUE data (line and continuum) relating to nuclear activity in your galaxies.

FILIPPENKO: Most of the objects have not been studied, but Drs C. Wu, T. Heckman, G. Reichert, and I are currently obtaining and analyzing $I U E$ data for a representative sample of LINERs. In general, extremely long integration times are required for spectra of sufficiently high quality. Goodrich and Keel (1986, Ap. J., 305, 148) recently looked at three LINERs; they detected a nonstellar continuum and He II $\lambda 1640$ in NGC 4579, but only starlight in NGC 5005 . The results for NGC 4501 were inconclusive because the galaxy is too faint. Bruzual, Peimbert, and Torres-Peimbert $(1982, A p . J ., 260,495)$ found an upper limit to the nonstellar UV continuum in M81 which is marginally consistent with the emission-line fluxes. No such radiation was detected in M87 by Fosbury et al. (1981, M. N. R. A. S., 197, 235). Ellis, Gondahalekar, and Efstathiou (1982, M. N. $R$. A. S., 201, 223) detected a photoionizing continuum in several nuclei, but argued that it is probably produced by hot young stars rather than by nonstellar processes. In all of these studies, very little mention was made of emission lines. We hope to explore the UV emission lines (and continua) more thoroughly, since they can be powerful diagnostics of the ionization mechanism. 